\title{
Acanthosis Nigricans in the Knuckles of Infants: A Novel Clinical Marker of High Metabolic Risk
}

\author{
René Rodríguez-Gutiérrez • Manuel E. de la O-Cavazos • \\ Alejandro Salcido-Montenegro • Adriana Sanchez-Garcia • \\ Minerva Gomez-Flores • Victoria Gonzalez-Nava • Dalia Castillo-Gonzalez • \\ Karla M. Santos-Santillana • José Gerardo González-González
}

Received: August 26, 2019 / Published online: October 10, 2019

(C) The Author(s) 2019

\begin{abstract}
Introduction: Acanthosis nigricans ( $\mathrm{AN})$ is an early clinical sign of insulin resistance (IR) primarily in adults. The prevalence and association of AN and IR in infants, however, remains uncertain. We aimed to describe the prevalence of $\mathrm{AN}$ and its association with IR in a group of Latin-American infants.
\end{abstract}

Enhanced Digital Features To view enhanced digital features for this article go to https://doi.org/10.6084/ m9.figshare.9891821.

R. Rodríguez-Gutiérrez · A. Salcido-Montenegro ·

A. Sanchez-Garcia · V. Gonzalez-Nava .

D. Castillo-Gonzalez - K. M. Santos-Santillana .

J. G. González-González (凶)

Endocrinology Division, Facultad de Medicina y

Hospital Universitario "Dr. Jose E. González",

Universidad Autonoma de Nuevo Leon, Ave.

Madero y Gonzalitos s/n, Colonia Mitras Centro, 64460 Monterrey, Nuevo León, Mexico

e-mail: jgerardo@meduanl.com

R. Rodríguez-Gutiérrez · A. Salcido-Montenegro ·

A. Sanchez-Garcia · V. Gonzalez-Nava .

D. Castillo-Gonzalez - K. M. Santos-Santillana .

J. G. González-González

Plataforma INVEST Medicina UANL-KER Unit Mayo

Clinic (KER Unit México), Universidad Autonoma

de Nuevo Leon, Av. Madero y Gonzalitos s/n,

Colonia Mitras Centro, 64460 Monterrey, Nuevo

León, Mexico

R. Rodríguez-Gutiérrez

Knowledge and Evaluation Research Unit in

Endocrinology, Mayo Clinic, Rochester, MN, USA
Methods: We studied a random sample of 227 healthy infants between 9 and 24 months of age. After a complete clinical history was obtained and a physical examination was performed, fasting plasma glucose and serum insulin were measured. Three blinded evaluators assessed $\mathrm{AN}$ in each patient. Infants with AN were categorized as cases. The HOMA-IR index cutoffs of $\geq 90$ th and $\geq 95$ th percentiles were considered IR.

Results: There were 49 infants with AN (21.6\%) (cases) and 178 without AN (78.4\%) (controls). Cases had a significantly higher mean serum

R. Rodríguez-Gutiérrez · J. G. González-González Research Unit, Facultad de Medicina y Hospital Universitario "Dr. Jose E. González", Universidad Autonoma de Nuevo Leon, Av. Madero y Gonzalitos s/n, Colonia Mitras Centro, 64460 Monterrey, Nuevo León, Mexico

M. E. de la O-Cavazos

Department of Pediatrics, Facultad de Medicina y Hospital Universitario "Dr. Jose E. González", Universidad Autonoma de Nuevo Leon, Av. Madero y Gonzalitos s/n, Colonia Mitras Centro, 64460 Monterrey, Nuevo León, Mexico

\section{Gomez-Flores}

Dermatology Division, Facultad de Medicina y Hospital Universitario "Dr. Jose E. González", Universidad Autonoma de Nuevo Leon, Av. Madero y Gonzalitos s/n, Colonia Mitras Centro, 64460 Monterrey, Nuevo León, Mexico 
insulin, fasting plasma glucose, and HOMA-IR levels of $3.67 \pm 2.56 \mu \mathrm{U} / \mathrm{ml}$ vs. $2.42 \pm 1.45 \mu \mathrm{U} /$ $\mathrm{ml}, P=0.005 ; 84.2 \pm 12.6 \mathrm{mg} / \mathrm{dL}$ vs. $77 \pm \mathrm{SD}$ $9.9 \mathrm{mg} / \mathrm{dL}, P \leq 0.001$; HOMA-IR $0.77 \pm 0.54$ vs. $0.46 \pm 0.28, P \leq 0.001$, respectively. More cases than controls presented HOMA-IR levels $\geq 95$ th percentile (cases 18.4\%; controls $0.5 \%, P$ $\leq 0.001$ ) and $\geq 90$ th percentile (cases $32.7 \%$; controls $1.6 \%, P \leq 0.001$ ). AN in the knuckles had a high sensitivity and a negative predictive value (NPV) for detecting patients with HOMAIR levels above the 95th percentile (sensitivity 90\%; NPV 99.4\%) and above the 90th percentile (sensitivity 84.2\%; NPV 98.3\%).

Conclusion: AN in the knuckles is a prevalent, non-invasive, costless, and reliable screening clinical tool that can be used for early detection of infants with IR and a high metabolic risk.

Keywords: Acanthosis nigricans; Hyperinsulinemia; Infants; Insulin resistance; Metabolic risk; Obesity

\section{INTRODUCTION}

In the last 25 years, pediatric obesity has doubled and is currently affecting more than 107 million children worldwide [1]. This will inevitably translate into an increase of the already unstoppable obesity and type 2 diabetes pandemic. Moreover, it would lead to a rise in chronic diabetes complications, including mortality, while at the same time worsening the high economic burden for the individual patient and any public health system [2-6]. In addition, many of these individuals will also suffer from other pathophysiological associated illnesses such as non-alcoholic fatty liver disease, polycystic ovarian syndrome, and some types of cancer [7-9]. Early detection of individuals at high metabolic risk and opportune lifestyle interventions are the most reliable measures to counter both epidemics $[2,3,10]$. Therefore, identifying clinical markers that could be used to recognize individuals at a high metabolic risk early in life would not only be desirable but also encouraging.

One of the earliest detectable pathophysiological abnormalities in the natural history of obesity and type 2 diabetes is insulin resistance (IR) [11-15]. The euglycemic insulin clamp is recognized as the gold standard to diagnose IR; however, it is expensive and technically difficult to perform. Several biochemical indexes such as HOMA-IR, QUICKI, and Matsuda have demonstrated a fairly acceptable diagnostic accuracy for IR, yet they are invasive and require fasting and a clinical indication to assess IR. Recently, numerous studies, primarily in adult populations, have demonstrated that acanthosis nigricans (AN) is an early clinical sign of IR $[16,17]$. In a young Latin-American cohort the knuckles were the most common site of AN, particularly in non-obese subjects and was positively associated with IR [17]. Identification of individuals with high metabolic risk much earlier in life would be ideal; however, it is not yet possible. There is a paucity of evidence, however, regarding the association of IR and AN in infants and studies have previously assessed AN only in the neck without a correlation with biochemical markers of IR [18-21]. Therefore, we conducted a cross-sectional comparative study in a group of Latin-American infants between 9 and 24 months of age with the aim of describing the prevalence of AN and determining its association with IR. Secondary objectives were to assess AN diagnostic accuracy for IR and to identify the presence of $\mathrm{AN}$ in different weight categories and their association with IR.

\section{METHODS}

\section{Study Participants}

Approval was obtained from the institutional review board and ethics committee of our university prior to data collection. From April 2016 to April 2017 we enrolled a consecutive and random sample of 227 healthy male and female infants between 9 and 24 months of age born at the "Dr. Jose E. Gonzalez" University Hospital. Parents and/or tutors were contacted via telephone to request their participation in the study. Infants were excluded from the study if they were taking any medication or had any illness that could impair insulin secretion or action, were critically ill, had a congenital 
disease, or if their parents or tutor refused to participate in the study. Infants younger than 9 months were arbitrarily excluded as most were not able to withstand the required 4-h fasting as a result of lactation or more frequent eating patterns. All parents or tutors provided informed consent before initiating the evaluation.

Infants who presented $\mathrm{AN}$ on the knuckles were categorized as cases. This group was further subdivided into patients with AN only in the knuckles (AN1), patients with $\mathrm{AN}$ in the knuckles in addition to other sites (neck, axillae, or elbows) (AN2), and AN in other sites but not on the knuckles (AN3). All participants who did not present $\mathrm{AN}$ on the knuckles or any other site were categorized as controls.

\section{Study Protocol}

Infants accompanied by their parents or tutors arrived to our institution at about 0800 hours, having at least a 4-h overnight fast. A comprehensive clinical history of each participant was obtained from the parents or tutor. Afterwards, participants underwent a complete physical examination that included anthropometric measures and a standardized close-up photography of the neck, axillae, elbows, and knuckles in order to evaluate and classify $\mathrm{AN}$ as previously described [22]. When available, biological mothers of the infants also underwent physical examination, including anthropometric measures and AN evaluation [22]. Afterwards, a single 6-ml blood sample was drawn to obtain plasma glucose and serum insulin levels.

\section{Measurements}

Participants' weight and height were determined on a calibrated Seca ${ }^{\circledR} 232$ and a stadiometer (TAQ Sistemas Médicos, S.A. de C.V., Mexico City, Mexico). Weight and height measurements of the participants' mothers were determined on a calibrated Seca ${ }^{\circledR} 700$ and a stadiometer (TAQ Sistemas Médicos, S.A. de C.V.). BMI was calculated using anthropometric measurements. Participants were classified as normal, overweight, and obese utilizing the sexspecific World Health Organization BMI for age birth to 2 years child growth standards [23]. Three experienced, independent, and blinded evaluators assessed AN presence in each patient. Concordance between the observers was evaluated by selecting a random sample of 30 patients. Disagreements between observers were resolved by consensus. Skin phototype was determined as previously reported [24]. Fasting plasma glucose determinations were assessed by the glucose oxidase method (Stat-Fax Spectrophotometer, Awareness Technology, Palm City Fl., intra-assay CV $1.4 \%$, inter-assay CV $0.6 \%$, and for fasting serum insulin, an electrochemiluminescence immunoassay (HitachiCobas e411, Roche, Mannheim, Germany, intraassay CV $\leq 2 \%$ ) was used. The HOMA-IR index was obtained as previously recommended [25].

Currently, there are no HOMA-IR index cutoff values reported for the age and ethnicity of our study population. Therefore, cutoff values of $\geq 90$ th and $\geq 95$ th percentiles were considered abnormal.

\section{Statistical Analysis}

To determine the sample size needed to measure AN prevalence, a pilot study with 40 infants was conducted in which the presence of $\mathrm{AN}$ was documented as $17 \%$. To compare HOMA-IR indexes between cases and controls at least 42 infants per group were required. Categorical variables are reported as percentages and frequencies; continuous variables are reported as means and standard deviations. Normality was determined using the Kolmogorov-Smirnov test. Categorical variables were compared using Pearson's $\chi^{2}$ test or Fisher's exact test for $2 \times 2$ tables. An unpaired Student's $t$ test or Mann-Whitney $U$ test were used to compare continuous variables according to normality. When more than two groups were compared, one-way ANOVA or the Kruskal-Wallis test was performed according to normality. The Bonferroni correction was used as a post hoc test. Interobserver reliability coefficients for AN assessment were calculated using Cohen's kappa. $P$ $\leq 0.05$ was considered statistically significant. IBM SPSS, Version 22.0 (IBM Corp., Armonk, NY) was used to perform the statistical analysis. 


\section{Statement of Ethics Compliance}

Approval was obtained from the institutional review board and ethics committee of our university, Universidad Autonoma de Nuevo Leon. Also, this study complied with the Helsinki Declaration of 1964, as revised in 2013, concerning human and animal rights, and the Springer policy concerning informed consent. All parents or tutors of the infants that participated in the study provided informed consent before enrolling in the study.

\section{RESULTS}

\section{Allocation Analysis and AN Prevalence}

A total of 227 infants were included in the study. Concordance coefficients between observers for the classification of AN in the neck, axillae, elbows, and knuckles are shown in Table 1. In all cases, Cohen's kappa (inter-observer reliability) was rated high. There were 49 infants with AN (21.6\%) and 178 controls (78.4\%). Among cases, 44 (19.3\%) were categorized as AN1, 5 (2.2\%) as AN2, and none as AN3 (Table 2).

\section{Study Population}

\section{Anthropometric and Demographic Characteristics}

Infants' mean age was $16.4 \pm 4.7$ months and $47.1 \%$ were female. More than $80 \%$ were classified as skin phototype 2 and 3 . Overweight and obesity were seen in $14.1 \%$ and $5.7 \%$, respectively. Overall, $57.3 \%$ of the study population had a family history of diabetes. There were no significant differences in the anthropometric, demographic, or perinatal characteristics between groups with the exception of a higher BMI in cases $(17.4 \pm 3.42)$ compared to controls $(16.47 \pm 2.28 ; P \leq 0.001)$ (Table 2).

\section{Biological Mothers' Characteristics}

Only 212/227 (93.4\%) biological mothers were able to attend the study consult and underwent physical exploration and AN assessment. Their mean age was $25.5 \pm 5.8$ years with a mean BMI of $27.35 \pm 6.52 \mathrm{~kg} / \mathrm{m}^{2}$. More than two-thirds of the biological mothers presented AN on any site (66\%). There was a major proportion of mothers with AN on any site in the cases group compared to controls, although it was not statistically significant $(77.3 \%$ vs. $63.1 \%$, respectively; $P=0.054)$. There was an overall prevalence of $4.8 \%$ of gestational diabetes mellitus (GDM) of the study participants' mothers. There were no differences in any of the mothers' characteristics between groups (Table 2).

\section{HOMA-IR Index Comparison}

Mean fasting insulin, glucose, and HOMA-IR values are shown in Table 3. Cases had a significant higher mean serum insulin level $(3.67 \pm 2.56 \mu \mathrm{U} / \mathrm{ml})$ when compared to controls $(2.42 \pm 1.45 \mu \mathrm{U} / \mathrm{ml}, P=0.005)$. This same trend was seen for fasting plasma glucose (cases $84.2 \pm 12.6 \mathrm{mg} / \mathrm{dL}$; controls $77 \pm 9.9 \mathrm{mg} / \mathrm{dL}$, $P \leq 0.001$ ) and HOMA-IR (cases $0.77 \pm 0.54$; controls $0.46 \pm 0.28, P \leq 0.001$ ). A major proportion of cases were above the 95th insulin percentile (cases 14.3\%; controls 2.2\%, $P$

Table 1 Concordance of acanthosis nigricans classification by clinical assessment

\begin{tabular}{|c|c|c|c|c|c|c|}
\hline \multirow{2}{*}{$N=\mathbf{3 0}$} & \multicolumn{2}{|l|}{ Observer 1 vs. 2} & \multicolumn{2}{|l|}{ Observer 1 vs. 3} & \multicolumn{2}{|l|}{ Observer 2 vs. 3} \\
\hline & Agreeing exactly (\%) & $\overline{\text { Kappa }}$ & Agreeing exactly (\%) & $\overline{\text { Kappa }}$ & Agreeing exactly (\%) & $\overline{\text { Kappa }}$ \\
\hline Neck & 90 & 0.78 & 96.6 & 0.93 & 93.3 & 0.86 \\
\hline Axillae & 83.3 & 0.66 & 90.0 & 0.80 & 80 & 0.6 \\
\hline Elbows & 93.3 & 0.86 & 93.3 & 0.86 & 96.6 & 0.93 \\
\hline Knuckles & 90.0 & 0.77 & 93.3 & 0.85 & 90.0 & 0.77 \\
\hline
\end{tabular}


Table 2 Demographic and anthropometric characteristics of the study population

\begin{tabular}{|c|c|c|c|c|}
\hline \multirow[t]{2}{*}{ Characteristic } & \multicolumn{3}{|c|}{ Acanthosis nigricans in the knuckles } & \multirow[t]{2}{*}{$P$ value } \\
\hline & $\begin{array}{l}\text { All patients } \\
(n=227)\end{array}$ & $\begin{array}{l}\text { Controls } \\
178(78.4)\end{array}$ & $\begin{array}{l}\text { Cases } \\
49(21.6)\end{array}$ & \\
\hline \multicolumn{5}{|l|}{ Patients' characteristics at time of visit } \\
\hline Mean age (rectified) (SD), months & $16.4( \pm 4.7)$ & $16.4( \pm 4.8)$ & $16.1( \pm 4.6)$ & 0.71 \\
\hline Female sex & $107(47.1)$ & $78(43.8)$ & $29(59.2)$ & 0.05 \\
\hline Mean weight (SD), $\mathrm{kg}$ & $10.5( \pm 1.63)$ & $10.38( \pm 1.56)$ & $10.93( \pm 1.82)$ & 0.05 \\
\hline Mean height (SD), m & $0.79( \pm 0.06)$ & $0.79( \pm 0.05)$ & $0.79( \pm 0.07)$ & 0.69 \\
\hline Mean BMI (SD), $\mathrm{kg} / \mathrm{m}^{2}$ & $16.47( \pm 2.24)$ & $16.22( \pm 1.74)$ & $17.38( \pm 3.39)$ & $<0.001$ \\
\hline \multicolumn{5}{|l|}{ BMI-for-age percentiles } \\
\hline$\leq 3$ & $13(5.7)$ & $13(7.3)$ & $0(0)$ & 0.09 \\
\hline 3 to $\leq 15$ & $28(12.3)$ & $23(12.9)$ & $5(10.2)$ & \\
\hline 15 to $\leq 50$ & $66(29.1)$ & $53(29.8)$ & $13(26.5)$ & \\
\hline 50 to $\leq 85$ & $73(32.2)$ & $55(30.9)$ & $18(36.7)$ & \\
\hline 85 to $\leq 97$ & $34(15)$ & $27(15.1)$ & $7(14.3)$ & \\
\hline$\geq 97$ & $13(5.7)$ & $7(3.9)$ & $6(12.2)$ & \\
\hline Fasting hours, mean (SD) & $6.23( \pm 3)$ & $6.3( \pm 3.06)$ & $5.97( \pm 2.81)$ & 0.46 \\
\hline Family history of diabetes & $130(57.3)$ & $105(59)$ & $25(51)$ & 0.31 \\
\hline \multicolumn{5}{|l|}{ BMI } \\
\hline Normal & $182(80.2)$ & $145(81.5)$ & $37(75.5)$ & 0.08 \\
\hline Overweight & $32(14.1)$ & $26(14.6)$ & $6(12.2)$ & \\
\hline Obese & $13(5.7)$ & $7(3.9)$ & $6(12.2)$ & \\
\hline \multicolumn{5}{|l|}{ Phototype } \\
\hline 2 & $81(35.7)$ & $66(37.1)$ & $15(30.6)$ & 0.10 \\
\hline 3 & $110(48.5)$ & $88(49.4)$ & $22(44.9)$ & \\
\hline 4 & $35(15.4)$ & $24(13.5)$ & $11(22.4)$ & \\
\hline 5 & $1(0.4)$ & $0(0)$ & $1(2)$ & \\
\hline AN1 & & & $44(89.8)$ & \\
\hline AN2 & & & $5(10.2)$ & \\
\hline \multicolumn{5}{|l|}{ Perinatal characteristics } \\
\hline Born to women with GDM & $11(4.8)$ & $10(5.6)$ & $1(2)$ & 0.30 \\
\hline \multicolumn{5}{|l|}{ Delivery } \\
\hline Vaginal & $125(57.9)$ & $98(58.3)$ & $27(56.3)$ & 0.86 \\
\hline Cesarean section & $91(42.1)$ & $70(41.7)$ & $21(43.8)$ & \\
\hline
\end{tabular}


Table 2 continued

\begin{tabular}{|c|c|c|c|c|}
\hline \multirow[t]{2}{*}{ Characteristic } & \multicolumn{3}{|c|}{ Acanthosis nigricans in the knuckles } & \multirow[t]{2}{*}{$P$ value } \\
\hline & $\begin{array}{l}\text { All patients } \\
(n=227)\end{array}$ & $\begin{array}{l}\text { Controls } \\
178(78.4)\end{array}$ & $\begin{array}{l}\text { Cases } \\
49(21.6)\end{array}$ & \\
\hline Preterm birth & $29(12.8)$ & $23(12.9)$ & $6(12.2)$ & 0.9 \\
\hline Mean gestational age (SD), weeks & $38.4( \pm 2.9)$ & $38.2( \pm 3)$ & $38.9( \pm 2.4)$ & 0.16 \\
\hline Mean birth weight (SD), g & $3026( \pm 626.9)$ & $3020.2( \pm 636)$ & $3047.7( \pm 597.5)$ & 0.83 \\
\hline Mean height, mean (SD), cm & $49.3( \pm 4.2)$ & $49.4( \pm 4.3)$ & $48.8( \pm 3.9)$ & 0.38 \\
\hline Breastfed & $204(90.2)$ & $158(89.3)$ & $46(93.9)$ & 0.54 \\
\hline Mean duration of breastfeed (SD), months & $7.60( \pm 6.16)$ & $7.35( \pm 6.2)$ & $8.5( \pm 5.99)$ & 0.23 \\
\hline Infant formula & $185(81.9)$ & $146(82.5)$ & $39(79.6)$ & 0.84 \\
\hline Mean duration using milk formula (SD), months & $9.7( \pm 7.2)$ & $9.8( \pm 7.2)$ & $9.1( \pm 7.4)$ & 0.46 \\
\hline \multicolumn{5}{|l|}{ Biological mothers' characteristics } \\
\hline Mean age (SD), year & $25.5( \pm 5.8)$ & $25.7( \pm 6)$ & $24.8( \pm 5.2)$ & 0.34 \\
\hline AN presence, any site & $140(66)$ & $106(63.1)$ & $34(77.3)$ & 0.05 \\
\hline GDM presence, any pregnancy & $16(7)$ & $15(8.4)$ & $1(2)$ & 0.12 \\
\hline Mean BMI (SD), $\mathrm{kg} / \mathrm{m}^{2}$ & $27.35( \pm 6.52)$ & $27.08( \pm 6.78)$ & $28.4( \pm 5.36)$ & 0.28 \\
\hline Metformin use during pregnancy & $5(2.2)$ & $5(2.8)$ & $0(0)$ & 0.23 \\
\hline Previous use of corticosteroids & $15(6.6)$ & $12(6.7)$ & $3(6.1)$ & 0.87 \\
\hline
\end{tabular}

Data are presented as numbers (percentages) of patients unless otherwise indicated

$B M I$ body mass index, $A N 1$ acanthosis nigricans in the knuckle only, $A N 2$ acanthosis nigricans in the knuckles and other site, GDM gestational diabetes mellitus

$\leq 0.001)$ and above the 90th insulin percentile (cases 30.6\%; controls 4.4\%, $P \leq 0.001$ ). Similar results were observed when both groups were compared by the glucose 95th percentile cutoff (cases 14.3\%; controls 6.1\%, $P \leq 0.001$ ) and 90th percentile cutoff (cases 22.4\%; controls $8.4 \%, P \leq 0.001)$. Finally, more cases than controls presented HOMA-IR levels $\geq 95$ th percentile (cases $18.4 \%$; controls $0.5 \%, P \leq 0.001$ ) and $\geq 90$ th percentile (cases $32.7 \%$; controls $1.6 \%, P \leq 0.001)$.

Table 4 compares the HOMA-IR between cases and controls allocated by BMI. The majority of cases and controls had a normal BMI (cases $75.5 \%$; controls $81.5 \%$ ) compared to the overweight (cases 12.2\%; controls 14.6\%) and obese categories (cases 12.2\%; controls 3.9\%, $P=0.084$ ) (Table 2). Cases with a normal BMI presented higher HOMA-IR levels than controls (cases $0.76 \pm 0.54 ; \quad$ controls $0.45 \pm 0.29$, $P=0.003)$. Although not statistically significant, this trend was also observed in overweight (cases $1.04 \pm 0.64 ; \quad$ controls $0.53 \pm 0.32$, $P=0.053$ ) and obese patients (cases $0.59 \pm 0.48$; controls $0.54 \pm 0.30, P=0.945$ ). A minor proportion of cases and controls with normal BMI presented HOMA-IR levels above percentile 95 (cases 19.9\%; controls $1.4 \%, P \leq 0.001$ ) and 90 (cases 29.8\%; controls 2.4\%, $P \leq 0.001$ ). This trend continued when overweight cases and controls were compared for HOMA-IR $\geq 95$ th percentile (cases 33\%; controls $0 \%, P=0.002$ ) and $\geq 90$ th percentile (cases $7.7 \%$; controls $50 \%, P=0.01$ ). However, in the obese group there were no differences in insulin or HOMA-IR in both the $\geq 95$ th and $\geq 90$ th percentiles. 
Table 3 Mean fasting serum insulin, glucose, and HOMA-IR values

\begin{tabular}{|c|c|c|c|}
\hline \multirow[t]{2}{*}{ Measurements characteristics } & \multicolumn{2}{|c|}{ Acanthosis nigricans in the knuckles } & \multirow[t]{2}{*}{$P$ value } \\
\hline & $\begin{array}{l}\text { Controls } \\
178(78.4)\end{array}$ & $\begin{array}{l}\text { Cases } \\
49(21.6)\end{array}$ & \\
\hline \multicolumn{4}{|l|}{ Insulin } \\
\hline Mean insulin (SD), $\mu \mathrm{U} / \mathrm{ml}$ & $2.42( \pm 1.45)$ & $3.67( \pm 2.56)$ & 0.005 \\
\hline \multicolumn{4}{|l|}{ Insulin percentile 95} \\
\hline$\leq 6.95$ & $174(97.7)$ & $42(85.7)$ & 0.001 \\
\hline$\geq 6.95$ & $4(2.2)$ & $7(14.3)$ & \\
\hline \multicolumn{4}{|l|}{ Insulin percentile 90} \\
\hline$\leq 5.34$ & $170(95.5)$ & $34(69.4)$ & $<0.001$ \\
\hline$\geq 5.34$ & $8(4.4)$ & $15(30.6)$ & \\
\hline \multicolumn{4}{|l|}{ Glucose } \\
\hline Mean glucose (SD), mg/dl & $77( \pm 9.9)$ & $84.2( \pm 12.6)$ & $<0.001$ \\
\hline \multicolumn{4}{|l|}{ Glucose mg/dl } \\
\hline$\leq 100$ & $175(98.3)$ & $43(87.8)$ & $<0.001$ \\
\hline$\geq 100$ & $3(1.6)$ & $6(12.2)$ & \\
\hline \multicolumn{4}{|l|}{ Glucose percentile 95} \\
\hline$\leq 97$ & $167(93.8)$ & $42(85.7)$ & 0.004 \\
\hline$\geq 97$ & $11(6.17)$ & $7(14.3)$ & \\
\hline \multicolumn{4}{|l|}{ Glucose percentile 90} \\
\hline$\leq 95$ & $163(91.5)$ & $38(77.6)$ & $<0.001$ \\
\hline$\geq 95$ & $15(8.4)$ & $11(22.4)$ & \\
\hline \multicolumn{4}{|l|}{ HOMA-IR } \\
\hline Mean HOMA-IR (SD) & $0.46( \pm 0.28)$ & $0.77( \pm 0.54)$ & 0.001 \\
\hline \multicolumn{4}{|l|}{ HOMA-IR percentile 95} \\
\hline$\leq 1.27$ & $177(99.4)$ & $40(81.6)$ & $<0.001$ \\
\hline$\geq 1.27$ & $1(0.5)$ & $9(18.4)$ & \\
\hline \multicolumn{4}{|l|}{ HOMA-IR percentile 90} \\
\hline$\leq 1.04$ & $175(98.3)$ & $33(67.3)$ & $<0.001$ \\
\hline$\geq 1.04$ & $3(1.6)$ & $16(32.7)$ & \\
\hline
\end{tabular}

Data are presented as numbers (percentages) of patients unless otherwise indicated

\section{Diagnostic Accuracy of AN for IR}

The sensitivity and specificity of using AN in the knuckles for detecting patients above the 95th and 90th percentile of HOMA-IR are shown in Table 5. The presence of AN in the knuckles had a high sensitivity and negative predictive value (NPV) for detecting patients with HOMA-IR 
Table 4 Mean fasting Insulin, Glucose and HOMA-IR values analyzed by BMI group

\begin{tabular}{|c|c|c|c|c|c|c|c|c|c|}
\hline \multirow[t]{3}{*}{ BMI } & \multirow[t]{3}{*}{$n$} & \multicolumn{8}{|l|}{ HOMA-IR } \\
\hline & & \multicolumn{2}{|c|}{ Mean HOMA-IR (SD) } & \multicolumn{3}{|c|}{ HOMA-IR $\geq 90$ th percentile } & \multicolumn{3}{|c|}{ HOMA-IR $\geq 95$ th percentile } \\
\hline & & & $P$ value & $<1.04$ & $\geq 1.04$ & $P$ value & $<1.270$ & $\geq 1.270$ & $P$ value \\
\hline \multicolumn{10}{|l|}{ Normal } \\
\hline Controls & 145 & $0.45( \pm 0.29)$ & 0.003 & $141(97.2)$ & $4(2.8)$ & $<0.001$ & $143(98.6)$ & $2(1.4)$ & $<0.001$ \\
\hline Cases & 37 & $0.76( \pm 0.54)$ & & $26(70.2)$ & $11(29.8)$ & & $30(81.1)$ & $7(19.9)$ & \\
\hline \multicolumn{10}{|l|}{ Overweight } \\
\hline Controls & 26 & $0.53( \pm 0.32)$ & 0.05 & $24(92.3)$ & $2(7.7)$ & 0.01 & $26(100)$ & $0(0)$ & 0.002 \\
\hline Cases & 6 & $1.04( \pm 0.64)$ & & $3(50)$ & $3(50)$ & & $4(66.7)$ & $2(33.3)$ & \\
\hline \multicolumn{10}{|l|}{ Obese } \\
\hline Controls & 7 & $0.54( \pm 0.30)$ & 0.94 & $7(100)$ & $0(0)$ & 0.09 & $7(100)$ & $0(0)$ & $\mathrm{N} / \mathrm{A}$ \\
\hline Cases & 6 & $0.59( \pm 0.48)$ & & $4(66.7)$ & $2(33.3)$ & & $6(100)$ & $0(0)$ & \\
\hline
\end{tabular}

Insulin

\begin{tabular}{|c|c|c|c|c|c|c|}
\hline Mean insulin (SD), $\mu \mathrm{U} / \mathrm{ml}$ & \multicolumn{3}{|c|}{ Insulin $\geq 90$ th percentile } & \multicolumn{3}{|c|}{ Insulin $\geq 95$ th percentile } \\
\hline$P$ value & $\begin{array}{l}\leq 5.34 \\
\mu \mathrm{U} / \mathrm{ml}\end{array}$ & $\begin{array}{l}\geq 5.34 \\
\mu U / \mathrm{ml}\end{array}$ & $P$ value & $\leq 6.95 \mu \mathrm{U} / \mathrm{ml}$ & $\begin{array}{l}\geq 6.95 \\
\mu \mathrm{U} / \mathrm{ml}\end{array}$ & $P$ value \\
\hline
\end{tabular}

Normal

\begin{tabular}{|c|c|c|c|c|c|c|c|c|}
\hline Controls & 145 & $2.37( \pm 1.55)$ & 0.009 & $140(96.6)$ & $5(3.4)$ & $<0.001$ & $143(98.6)$ & $2(1.4)$ \\
\hline Cases & 37 & $3.69( \pm 2.61)$ & & $25(67.6)$ & $12(32.4)$ & & $32(86.5)$ & $5(13.5)$ \\
\hline
\end{tabular}

Overweight

\begin{tabular}{|c|c|c|c|c|c|c|c|c|}
\hline Controls & 26 & $2.7( \pm 1.59)$ & 0.05 & $25(96.2)$ & $1(3.8)$ & 0.002 & $26(100)$ & $0(0)$ \\
\hline Cases & 6 & $5.01( \pm 2.62)$ & & $3(50)$ & $3(50)$ & & $4(66.7)$ & $2(33.3)$ \\
\hline
\end{tabular}

Obese

\begin{tabular}{|c|c|c|c|c|c|c|c|c|}
\hline Controls & 7 & $2.84( \pm 1.37)$ & 0.62 & $7(100)$ & $0(0)$ & $\mathrm{N} / \mathrm{A}$ & $7(100)$ & $0(0)$ \\
\hline Cases & 6 & $2.26( \pm 1.59)$ & & $6(100)$ & $0(0)$ & & $6(100)$ & $0(0)$ \\
\hline
\end{tabular}

\begin{tabular}{lllll}
$\frac{\text { Glucose }}{\text { Mean glucose (SD), } \mathrm{mg} / \mathrm{dl}}$ & & Glucose $\geq 90$ th percentile & Glucose $\geq 95$ th percentile \\
\hline$P$ value & $\begin{array}{l}\leq 94 \\
\mathrm{mg} / \mathrm{dl}\end{array}$ & $\geq 94 \mathrm{mg} / \mathrm{dl} \quad P$ value & $\leq 97 \mathrm{mg} / \mathrm{dl} \geq 97 \mathrm{mg} / \mathrm{dl} \quad P$ value \\
\hline
\end{tabular}

Normal

\begin{tabular}{|c|c|c|c|c|c|c|c|c|}
\hline Controls & 145 & $76.5( \pm 9.9)$ & 0.005 & 136 (93.7) & $9(6.3)$ & 0.04 & $140(96.6)$ & $5(3.4)$ \\
\hline Cases & 37 & $82.1( \pm 10.3)$ & & $31(83.8)$ & $6(16.2)$ & & $35(94.6)$ & $2(5.4)$ \\
\hline
\end{tabular}

Overweight

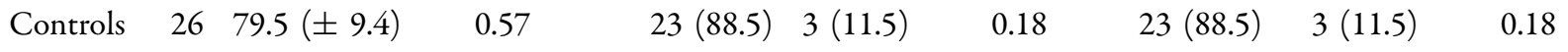


Table 4 continued

\begin{tabular}{|c|c|c|c|c|c|c|c|c|c|}
\hline & & \multicolumn{8}{|l|}{ Glucose } \\
\hline & & \multicolumn{2}{|c|}{ Mean glucose $(\mathrm{SD}), \mathrm{mg} / \mathrm{dl}$} & \multicolumn{3}{|c|}{ Glucose $\geq 90$ th percentile } & \multicolumn{3}{|c|}{ Glucose $\geq 95$ th percentile } \\
\hline & & & $P$ value & $\begin{array}{l}\leq 94 \\
\mathrm{mg} / \mathrm{dl}\end{array}$ & $\geq 94 \mathrm{mg} / \mathrm{dl}$ & $P$ value & $\leq 97 \mathrm{mg} / \mathrm{dl}$ & $\geq 97 \mathrm{mg} / \mathrm{dl}$ & $P$ value \\
\hline Cases & 6 & $83.2( \pm 15.4)$ & & $4(66.7)$ & $2(33.3)$ & & $4(66.7)$ & $2(33.3)$ & \\
\hline \multicolumn{10}{|l|}{ Obese } \\
\hline Controls & 7 & $76.6( \pm 8)$ & 0.008 & $7(100)$ & $0(0)$ & 0.03 & $7(100)$ & $0(0)$ & 0.03 \\
\hline Cases & 6 & $98.5( \pm 15.3)$ & & $3(50)$ & $3(50)$ & & $3(50)$ & $3(50)$ & \\
\hline
\end{tabular}

Data are presented as numbers (percentages) of patients unless otherwise indicated

$B M I$ body mass index, $N / A$ not applicable

Table 5 Sensitivity and specificity of acanthosis nigricans as a marker for HOMA-IR detection

\begin{tabular}{|c|c|c|c|c|}
\hline & \multicolumn{2}{|l|}{ Overall } & \multicolumn{2}{|l|}{ Overall } \\
\hline & $\begin{array}{l}\text { HOMA IR > 95th } \\
\text { percentile }\end{array}$ & $\begin{array}{l}\text { HOMA IR }<95 \text { th } \\
\text { percentile }\end{array}$ & $\begin{array}{l}\text { HOMA IR }>90 \text { th } \\
\text { percentile }\end{array}$ & $\begin{array}{l}\text { HOMA IR < 90th } \\
\text { percentile }\end{array}$ \\
\hline Cases & 9 & 40 & 16 & 33 \\
\hline Controls & 1 & 177 & 3 & 175 \\
\hline Sensitivity & 90 & & 84.2 & \\
\hline Specificity & & 81.5 & & 84.2 \\
\hline $\begin{array}{l}\text { Positive predictive } \\
\text { value }\end{array}$ & 18.4 & & 32.6 & \\
\hline $\begin{array}{l}\text { Negative predictive } \\
\text { value }\end{array}$ & 99.4 & & 98.3 & \\
\hline
\end{tabular}

levels above the 95th percentile (sensitivity 90\%; NPV 99.4\%) and 90th percentile (sensitivity $84.2 \%$; NPV $98.3 \%$ ). The positive predictive value and specificity of AN was higher when detecting HOMA-IR values $\geq 90$ th percentile $(32.6 \%$ and $84.2 \%$, respectively) than $\geq 95$ th percentile $(18.4 \%$ and $81.5 \%$, respectively).

\section{DISCUSSION}

In this large observational study of infants between 9 and 24 months old, we found that one out of every five infants has AN.
Interestingly, $\mathrm{AN}$ in the knuckles was positive in all cases and only a minority of patients $(2.2 \%)$ had $\mathrm{AN}$ in other more classical sites (i.e., neck, axillae). Infants with AN were significantly associated with a higher HOMA-IR, fasting plasma glucose, and serum insulin levels; and to our knowledge, for the first time in the literature, we reliably report $\mathrm{a} \geq 90$ th $(\geq 1.04)$ and $\geq 95$ th $(\geq 1.27)$ percentile cutoff for HOMA-IR in infants. AN in the knuckles had a high sensitivity (84-90\%) and negative predictive value (98-99\%) for both $\geq 90$ th and $\geq$ 95th HOMA-IR percentiles.

Previous studies have assessed AN in children at age 2 years and older and have reported a 
range of $4.7-19 \%$ with a higher prevalence in overweight and obese children (up to 55\%) [20, 26-28]. Nevertheless, these studies often limit their assessment of AN to the neck, with a lack of a high inter-observer reliability. Moreover, most of these did not evaluate its association with biochemical markers of IR. The prevalence of AN in our study population was high $(21.6 \%)$ with the knuckles being the main contributor to this result. When the knuckles and other sites were considered, AN prevalence was only $2.2 \%$. This finding resonates with previous literature in which, in a healthy population of young Latino adults (18-23 years old), the knuckles were the most prevalent site of $\mathrm{AN}$; hence, this suggests that $\mathrm{AN}$ in the knuckles might be one of the earliest clinical signs of IR (however, often ignored because of the false notion that the neck or axillae are more common sites) and of paramount importance to direct efforts to the most needed (i.e., individuals at high metabolic risk) in whom early implementation of lifestyle changes could have a profound impact [15-17]. This finding is also supported by the fact that AN seems to progress with time as the prevalence found at ages 18-23 years was around 50\% (compared to $21 \%$ in our study) [16]. Hence, exclusion of the knuckles may delay the clinical diagnosis of IR until its appearance in classical and well-known locations of AN (i.e., the neck or axillae), thereby losing invaluable time to start management of IR.

To date, the association between AN presence and IR in children has been scarcely studied. Kobaissi et al. [29] aimed to describe the clinical value of $\mathrm{AN}$ as a predictor of insulin sensitivity in overweight Hispanic children (8-13 years), concluding that BMI variation was the main predictor of insulin sensitivity, whereas the presence of AN explained only 4\% of the estimated variation. Yet children were reported (by a single observer) with a high prevalence of AN (73.3\%). The exclusion of patients with normal BMI limits the applicability of AN to a very specific population [29]. In contrast, our study evaluated with high interobserver reliability the value of $\mathrm{AN}$ as a marker of IR at age 2 or younger, independently of their BMI, ensuring its applicability in a boarder range of individuals. Furthermore, we found higher HOMA-IR index values and basal serum insulin levels in participants with $\mathrm{AN}$ in the knuckles compared to controls. This suggests that $\mathrm{AN}$ is an early and reliable clinical sign to detect patients at high metabolic risk prior to any other usual clinical manifestations (e.g., weight gain, hyperglycemia, hypertension).

Our reported high sensitivity (84-90\%) and negative predictive value ( $>98 \%$ ) of AN on the knuckles set this clinical sign as an accurate, non-invasive, and straightforward marker of IR early in life. In perspective, if $\mathrm{AN}$ on the knuckles is not detected in a child, he or she would have a greater than $98 \%$ probability of not having IR using both HOMA-IR 90th and 95th percentiles cutoff values. On the other hand, according to our AN prevalence (21.6\%) and with our reported diagnostic performance, around $80 \%$ of the total population at this early stage of life could reliably and easily be recognized as not having IR.

Until now, few studies have described the HOMA-IR index in children and reliable cutoff values have not been widely accepted. Borgoño et al. [18] compared the HOMA-IR index of 1-year-old children born from mothers with and without GDM and concluded that for infants born from women with GDM, weight gain was positively associated with IR. In contrast to the results of Borgoño et al., the HOMA-IR index values reported in our study were higher $(0.21$ vs. 0.53 , respectively). However, their population consisted mostly of Caucasian patients (75\%) and non-specified race/ethnicity (25\%) [18]. Besides, the different insulin and glucose laboratory techniques could explain this variation. In fact, compared to Caucasians, higher HOMA-IR index cutoff values have been suggested for Latin-American population [30]. Therefore, as a result of the paucity of evidence, we opted to define the HOMA-IR index cutoff value by percentile and reliably describe the 90th and 95th percentiles.

Our study has several limitations. First, as a result of the study's design, we cannot accurately predict how higher HOMA-IR or high insulin values will impact infants later in life. However, even though this was not the aim of this study, in an ongoing study, we will follow 
the participants of this study and assess their complete evolution through time. In fact, the body of evidence is strong regarding the association between metabolic risk and higher HOMA-IR, insulin, and glucose values. Second, we used HOMA-IR to define IR; however, as a result of our large study sample, performing a euglycemic insulin clamp would not have been feasible because of its technical complexity. Moreover, the euglycemic insulin clamp has not been completely validated in this population. At the same time, we followed a strict protocol and, to our knowledge, this is the first study in a large number of infants with a high inter-observer reliability to evaluate AN prevalence, its association with IR, and AN diagnostic accuracy for IR.

\section{CONCLUSION}

More than one in five infants aged 9-24 months have AN in the knuckles and its presence is positively associated with IR. Owing to its high sensitivity and negative predictive value, AN in the knuckles is a non-invasive, easy-to-perform, costless, accurate, and reliable screening clinical tool that can be used for early detection of infants with IR and at high metabolic risk. This could translate into a timely, effective, and feasible way to direct preventive strategies to young individuals at high metabolic risk.

\section{ACKNOWLEDGEMENTS}

Funding. No funding or sponsorship was received for this study or publication of this article. The Rapid Service Fee was funded by the authors.

Editorial Assistance. We would like to thank Dr. Sergio Lozano-Rodriguez from the Research Division of the University (Universidad Autonoma de Nuevo Leon) for his critical reading, editing, and counseling of the manuscript. No funding was provided for this assistance.
Authorship. All named authors meet the International Committee of Medical Journal Editors (ICMJE) criteria for authorship for this article, take responsibility for the integrity of the work as a whole, and have given their approval for this version to be published.

Disclosures. René Rodríguez-Gutiérrez, Manuel E. de la O-Cavazos, Alejandro SalcidoMontenegro, Adriana Sanchez-Garcia, Minerva Gomez-Flores, Victoria Gonzalez-Nava, Dalia Castillo-Gonzalez, Karla M. Santos-Santillana, and José Gerardo González-González have nothing to disclose.

Compliance with Ethics Guidelines. Approval was obtained from the institutional review board and ethics committee of our university, Universidad Autonoma de Nuevo Leon. Also, this study complied with the Helsinki Declaration of 1964, as revised in 2013, concerning human and animal rights, and the Springer policy concerning informed consent. All parents or tutors of the infants that participated in the study provided informed consent before enrolling in the study.

Data Availability. The datasets generated and analyzed during the current study are available from the corresponding author on reasonable request.

Open Access. This article is distributed under the terms of the Creative Commons Attribution-NonCommercial 4.0 International License (http://creativecommons.org/licenses/ by-nc/4.0/), which permits any noncommercial use, distribution, and reproduction in any medium, provided you give appropriate credit to the original author(s) and the source, provide a link to the Creative Commons license, and indicate if changes were made.

\section{REFERENCES}

1. GBD 2015 Obesity Collaborators, Afshin A, Forouzanfar M, et al. Health effects of overweight and obesity in 195 countries over 25 years. N Engl J Med. 2017;377:13-27. 
2. Global BMI Mortality Collaboration, Di Angelantonio E, Bhupathiraju S, et al. Body-mass index and all-cause mortality: individual-participant-data meta-analysis of 239 prospective studies in four continents. Lancet. 2016;388:766-86.

3. Bommer C, Heesemann E, Sagalova V, et al. The global economic burden of diabetes in adults aged 20-79 years: a cost-of-illness study. Lancet Diabetes Endocrinol. 2017;5:423-30.

4. Shah AD, Langenberg C, Rapsomaniki E, et al. Type 2 diabetes and incidence of cardiovascular diseases: a cohort study in 1.9 million people. Lancet Diabetes Endocrinol. 2015;3:105-13.

5. Hubert HB, Feinleib M, McNamara PM, Castelli WP. Obesity as an independent risk factor for cardiovascular disease: a 26-year follow-up of participants in the Framingham Heart Study. Circulation. 1983;67:968-77.

6. Tancredi M, Rosengren A, Svensson A-M, et al. Excess mortality among persons with type 2 diabetes. N Engl J Med. 2015;373:1720-32.

7. Apridonidze T, Essah PA, Iuorno MJ, Nestler JE. Prevalence and characteristics of the metabolic syndrome in women with polycystic ovary syndrome. J Clin Endocrinol Metab. 2005;90:1929-35.

8. Renehan AG, Tyson M, Egger M, Heller RF, Zwahlen $M$. Body-mass index and incidence of cancer: a systematic review and meta-analysis of prospective observational studies. Lancet. 2008;371:569-78.

9. Younossi Z, Anstee QM, Marietti M, et al. Global burden of NAFLD and NASH: trends, predictions, risk factors and prevention. Nat Rev Gastroenterol Hepatol. 2018;15:11-20.

10. Murray CJL, Phil D, Lopez AD. Measuring the global burden of disease. N Engl J Med. 2013;369:448-57.

11. González-Saldivar G, Rodríguez-Gutiérrez R, Ocampo-Candiani J, González-González JG, Gómez-Flores M. Skin manifestations of insulin resistance: from a biochemical stance to a clinical diagnosis and management. Dermatol Ther (Heidelb). 2017;7:37-51.

12. Festa A, Williams K, D'Agostino R, Wagenknecht LE, Haffner SM. The natural course of $\beta$-cell function in nondiabetic the insulin resistance atherosclerosis study. Diabetes. 2006;55:1114-20.

13. Viner RM, Segal TY, Lichtarowicz-Krynska E, Hindmarsh P. Prevalence of the insulin resistance syndrome in obesity. Arch Dis Child. 2005;90:10-4.

14. Lorenzo C, Wagenknecht LE, D'Agostino RB, Rewers MJ, Karter AJ, Haffner SM. Insulin resistance, $\beta$-cell dysfunction, and conversion to type 2 diabetes in a multiethnic population. The insulin resistance atherosclerosis study. Diabetes Care. 2010;33:67-72.

15. Rodríguez-Gutiérrez R, Salcido-Montenegro A, González-González JG. Early clinical expressions of insulin resistance: the real enemy to look for. Diabetes Ther. 2018;9:435-8.

16. Gómez-Flores M, González-Saldivar G, Santos-Santos $\mathrm{O}$, et al. Implications of a clinically ignored site of acanthosis nigricans: the knuckles. Exp Clin Endocrinol Diabetes. 2015;123:27-33.

17. González-Saldivar G, Rodríguez-Gutiérrez R, Treviño-Alvarez AM, et al. Acanthosis nigricans in the knuckles: an early, accessible, straightforward, and sensitive clinical tool to predict insulin resistance. Dermatoendocrinology. 2018;10:1-29.

18. Borgoño CA, Hamilton JK, Ye C, et al. Determinants of insulin resistance in infants at age 1 year: impact of gestational diabetes mellitus. Diabetes Care. 2012;35:1795-7.

19. Veena SR, Krishnaveni GV, Willls AK, Hill JC, Karat SC, Fall CHD. Glucose tolerance and insulin resistance in indian children: relationship to infant feeding pattern. Diabetologia. 2011;54:2533-7.

20. Novotny R, Li F, Fialkowski MK, et al. Prevalence of obesity and acanthosis nigricans among young children in the children's healthy living program in the United States Affiliated Pacific. Medicine. 2016;95:6-11.

21. Kong AS, Vanderbloemen L, Skipper B, et al. Acanthosis nigricans predicts the clustering of metabolic syndrome components in Hispanic elemenatry school-aged children. J Pediatr Endocrinol Metab. 2012;25:1095-102.

22. Burke JP, Hale DE, Hazuda HP. A quantitative scale of acanthosis nigricans. Diabetes Care. 1999;22:1655-9.

23. Child growth standards BMI-for-age. 2017. http:// www.who.int/childgrowth/standards/bmi_for_age/ en/. Accessed 1 May 2017.

24. Fitzpatrick T. The validity and practicality of sunreactive skin types I through VI. Arch Dermatol. 1988;124:869-71.

25. Matthews DR, Hosker JP, Rudenski AS, Naylor BA, Treacher DF, Turner RC. Homeostasis model assessment: insulin resistance and beta-cell function from fasting plasma glucose and insulin concentrations in man. Diabetologia. 1985;28:412-9.

26. Martínez-Rojano H, Pizano-Zárate ML, SánchezJiménez B, Sámano R, López-Portillo A. Acantosis 
nigricans is associated with risk factors related to cardiovascular disease in Mexican children with obesity. Nutr Hosp. 2016;33:1081-90.

27. Kluczynik C, Mariz L, Souza L, Solano GB, Albuquerque F, Medeiros C. Acanthosis nigricans and insulin resistance in overweight children and adolescents. An Bras Dermatol. 2012;87:531-7.

28. Kong AS, Williams RL, Smith M, et al. Acanthosis nigricans and diabetes risk factors: prevalence in young persons seen in southwestern US primary care practices. Ann Fam Med. 2007;5:202-8.
29. Kobaissi HA, Weigensberg MJ, Ball GD, Cruz ML, Shaibi GQ, Goran MI. Relation between acanthosis nigricans and insulin sensitivity in overweight hispanic children at risk for type 2 diabetes. Diabetes Care. 2004;27:1412-6.

30. Dong B, Arnold LW, Peng Y, Wang Z. Ethnic differences in cardiometabolic risk among adolescents across the waist-height ratio spectrum: National Health and Nutrition Examination Surveys (NHANES). Int J Cardiol. 2016;222:622-8. 